\title{
Physicians' Perspectives Regarding Prescription Drug Monitoring Program Use Within the Department of Veterans Affairs: a Multi-State Qualitative Study
}

\author{
Thomas R. Radomski, MD, MS ${ }^{1,2,3}$, Felicia R. Bixler, MS', Susan L. Zickmund, PhD , \\ Katielynn M. Roman, $B A^{7}$, Carolyn T. Thorpe, $P h D, M P H^{1,5}$, Jennifer A. Hale, $B S^{7}$, \\ Florentina E. Sileanu, $M S^{7}$, Leslie R. M. Hausmann, $P h D^{7,2}$, Joshua M. Thorpe, $P h D, M P H^{1,5}$, \\ Katie J. Suda, PharmD, MS $S^{6}$, Kevin T. Stroupe, PhD ${ }^{6}$, Adam J. Gordon, MD, MPH', \\ Chester B. Good, MD, MPH $H^{1,2,5}$, Michael J. Fine, MD, MSc $c^{1,2}$, and Walid F. Gellad, MD, MPH $H^{1,2}$ \\ ${ }^{1}$ Center for Health Equity Research and Promotion, VA Pittsburgh Healthcare System, Pittsburgh, PA, USA; ${ }^{2}$ Division of General Internal Medicine, \\ Department of Medicine, University of Pittsburgh School of Medicine, Pittsburgh, PA, USA; ${ }^{3}$ Center for Research on Healthcare, Pittsburgh, PA, USA; \\ ${ }^{4}$ VA Salt Lake City Healthcare System, Salt Lake City, UT, USA; ${ }^{5}$ Department of Pharmacy and Therapeutics, University of Pittsburgh School of \\ Pharmacy, Pittsburgh, PA, USA; ${ }^{6}$ Edward Hines Jr. VA Hospital, Hines, IL, USA.
}

BACKGROUND: The Department of Veterans Affairs (VA) has implemented robust strategies to monitor prescription opioid dispensing, but these strategies have not accounted for opioids prescribed by non-VA providers. State-based prescription drug monitoring programs (PDMPs) are a potential tool to identify VA patients' receipt of opioids from non-VA prescribers, and recent legislation requires their use within VA.

OBJECTIVE: To evaluate VA physicians' perspectives and experiences regarding use of PDMPs to monitor Veterans' receipt of opioids from non-VA prescribers.

DESIGN: Qualitative study using semi-structured interviews.

PARTICIPANTS: Forty-two VA primary care physicians who prescribed opioids to 15 or more Veterans in 2015. We sampled physicians from two states with PDMPs (Massachusetts and Illinois) and one without prescriber access to a PDMP at the time of the interviews (Pennsylvania).

APPROACH: From February to August 2016, we conducted semi-structured telephone interviews that addressed the following topics regarding PDMPs: overall experiences, barriers to optimal use, and facilitators to improve use.

KEY RESULTS: VA physicians broadly supported use of PDMPs or desired access to one, while exhibiting varying patterns of PDMP use dictated by state laws and their clinical judgment. Physicians noted administrative burdens and incomplete or unavailable prescribing data as key barriers to PDMP use. To facilitate use, physicians endorsed (1) linking PDMPs with the VA electronic health record, (2) using templated notes to document PDMP use, and (3) delegating routine PDMP queries to ancillary staff. CONCLUSIONS: Despite the time and administrative burdens associated with their use, VA physicians in our study broadly supported PDMPs. The application of our findings

$\overline{\text { Electronic supplementary material The online version of this article }}$ (https://doi.org/10.1007/s11606-018-4374-1) contains supplementary material, which is available to authorized users.

Received July 6, 2017

Revised November 27, 2017

Accepted February 13, 2018

Published online March 8, 2018 to ongoing PDMP implementation efforts may strengthen PDMP use both within and outside VA and improve the safe prescribing of opioids.

KEY WORDS: Veterans; opioids; qualitative research; prescription drug monitoring programs.

J Gen Intern Med 33(8):1253-9

DOI: $10.1007 / \mathrm{s} 11606-018-4374-1$

(c) Society of General Internal Medicine (outside the USA) 2018

\section{BACKGROUND}

Opioid-related overdose and death has increased exponentially in the USA. ${ }^{1}$ Since 1999, overdose deaths from opioids have quadrupled, and unintentional overdoses now surpass motor vehicle accidents as the leading cause of injury death among Americans. ${ }^{1-3}$ Within the Department of Veterans Affairs (VA), opioid use has paralleled national trends, as the number of Veterans who received an opioid prescription from VA increased from 350,000 in 2003 to nearly 900,000 in 2013. ${ }^{4}$

VA has taken a multi-faceted approach to combat opioid misuse and abuse. Launched in 2012, the VA Opioid Safety Initiative (OSI) has sought to ensure safe opioid use through patient and prescriber education, close patient monitoring, and by increasing the availability of alternative pain therapies. ${ }^{4}$ While opioids prescribed within VA have decreased by $25 \%$ from their peak in 2013, Veterans continue to receive opioids from non-VA providers. ${ }^{4-8}$ In a cohort of Veterans enrolled in VA and Medicare Part D, more than one in eight received opioids from both systems in $2012 .{ }^{6}$ Moreover, the receipt of opioids from both VA and non-VA systems was associated with significantly increased risk of receiving opioids at potentially unsafe doses (> 120 morphine milligram equivalents per day) for 90 days or more. ${ }^{6}$ As dual use of VA and non-VA care continues to grow, an increasing number of Veterans may turn outside VA to receive opioids, thus undermining VA's internal efforts to reduce opioid-related overdose and death. ${ }^{9-13}$ 
State-based prescription drug monitoring programs (PDMPs) track dispensed controlled substances, including opioids, and are a tool to identify Veterans' receipt of opioids from non-VA prescribers. ${ }^{14-16}$ With the exception of Missouri, every state had an operational PDMP of some form as of 2016, although laws governing prescriber accessibility and use varied widely. ${ }^{15,16}$ Since receiving legislative approval in 2013, VA has promoted the use of PDMPs as part of the OSI, and has begun sharing pharmacy records with state PDMPs. However, studies conducted outside VA suggest a lack of physician buyin to PDMP use, due largely to administrative burdens and difficulty assessing up-to-date data. ${ }^{17-22}$ Recent federal legislation including the Comprehensive Addiction and Recovery Act and the Appropriations Bill of 2016 requires that VA implement CDC guidelines for safe opioid use, including PDMP use. ${ }^{23,24}$ Therefore, it is critical to ensure VA physician buy-in to conform with these mandates and optimally integrate the use of PDMPs into routine practice. Thus, our objective was to evaluate VA primary care physicians' perspectives and experiences regarding the use of PDMPs to monitor Veterans' use of opioids from non-VA sources.

\section{METHODS}

\section{Study Design and Sample}

We conducted a qualitative study of VA primary care physicians from February to August 2016 using open-ended questions in semi-structured interviews. We chose this approach to capture physicians' nuanced opinions about PDMPs that would otherwise be difficult to measure using closed-ended questions or quantitative survey methods.

We partnered with VA Pharmacy Benefits Management, who used administrative data to identify all VA primary care attending physicians who prescribed opioids to at least $15 \mathrm{VA}$ patients during fiscal year 2015. To highlight state-level differences in PDMPs, we purposefully sampled VA physicians who practiced in Illinois, Massachusetts, or Pennsylvania, because each of these states operated PDMPs with varying degrees of physician accessibility and laws governing use. ${ }^{23}$ For example, in Massachusetts, physicians were required to check the PDMP when issuing a new opioid prescription, whereas in Illinois, use was optional for such prescribing. At the time of this study, PDMP use in Pennsylvania was exclusively reserved for law enforcement personnel and not accessible to physicians.

We invited 317 physicians (64 from MA, 109 from IL, and 144 from PA) to participate in the study via email and sent email reminders to non-responders approximately 1 and 2 weeks after our first contact. Physicians who expressed interest and endorsed having cared for Veterans who received opioids from VA and non-VA prescribers were eligible for participation. There were 81 physicians that responded to our inquiry, of whom 22 declined to participate, 14 expressed interest but were lost to follow-up, and 3 were interested but were not eligible. A total of 42 physicians completed the interview.

\section{Data Collection (Interviews)}

An experienced interviewer trained in qualitative research methods conducted semi-structured telephone interviews using an interview script that was designed to take approximately 30 minutes to administer. The script broadly addressed topics regarding the dual use of opioids from VA and non-VA prescribers, with an emphasis on PDMP use (see Online Appendix). It contained open-ended questions and probes to extract additional detail as needed. The script was developed by the research team, refined by the qualitative expert (SZ), and pilot tested with two VA primary care physicians similar to those in the study. The final interview script and study protocol were approved by the Institutional Review Board of the VA Pittsburgh Healthcare System. Participants were consented to be audio-recorded prior to the interviews. Audio recordings were subsequently transcribed verbatim and verified to ensure accuracy.

\section{Codebook Development and Data Analysis}

Two trained qualitative analysts, including the interviewer, applied an open and iterative process to develop and refine the codebook using the standard approach by Crabtree and Miller. ${ }^{25-28}$ After systematically defining the codes, each coder applied the codebook across the interview, capturing relevant quotations. Each analyst independently coded approximately $20 \%$ of the transcripts using the Atlas.ti (Scientific Software, Berlin, Germany, version 7.5.11) program. We assessed inter-coder reliability, after which the coders applied an adjudication process to resolve any coding discrepancies. Given adequate inter-coder reliability (kappa $=0.70$ ), one coder subsequently coded the remaining transcripts independently. Based on the iterative codes that emerged from the coding process, we focused the analysis on three overarching thematic areas regarding PDMP use: (1) physicians' general experiences, (2) barriers to optimal PDMP use, and (3) facilitators of PDMP use. Within these areas, we also identified key differences between states when present.

\section{RESULTS}

\section{Participants}

Overall, 42 VA primary care physicians ( 12 from MA, 15 from IL, and 15 from PA) participated in the study (Table 1). Just over half of participants $(n=22 ; 52 \%)$ were male and 26 $(62 \%)$ were non-Hispanic white. Overall, participants practiced medicine following residency for a median of 18 years (IQR 10-24), with a median of 8 years (IQR 3-14) practicing within VA.

\section{Physicians' General Experiences}

Broad Acceptance but Varied Approaches to Use. Physicians from Illinois and Massachusetts were broadly supportive of PDMPs, and physicians from Pennsylvania generally desired 
Table 1Characteristics of Department of Veterans Affairs (VA) Primary Care Physician Respondents

\begin{tabular}{|c|c|c|c|c|}
\hline Characteristics & Overall respondents $(N=42)$ & Illinois $(N=15)$ & Massachusetts $(N=12)$ & Pennsylvania $(N=15)$ \\
\hline Gender, $n$ (\% female) & $20(48)$ & $8(53)$ & $7(58)$ & 5 (33) \\
\hline \multicolumn{5}{|l|}{ Race, $n(\%)$} \\
\hline Non-Hispanic white & $26(62)$ & $6(40)$ & $10(83)$ & $10(67)$ \\
\hline Other & $16(38)$ & $9(60)$ & $2(17)$ & $5(33)$ \\
\hline Years in practice, median $(\mathrm{B} 1-\mathrm{B} 3)$ & $18(10-24)$ & $12(8-22)$ & $20(6-31)$ & $18(12-20)$ \\
\hline Years practicing in VA, median (Q1-Q3) & $8(3-14)$ & $5.5(3-9)$ & $6(4-13.5)$ & $8(3-15)$ \\
\hline Clinical half days, median (Q1-Q33) & $7(4-9)$ & $8(4-9)$ & $6(4-8)$ & $7(4-9)$ \\
\hline \multicolumn{5}{|l|}{ Practice setting, $n(\%)^{*}$} \\
\hline VA medical center & $28(67)$ & $9(60)$ & $6(50)$ & $13(87)$ \\
\hline Community-based outpatient clinic & $15(36)$ & $6(40)$ & $7(58)$ & $2(13)$ \\
\hline Also practice outside VA, $n(\%)$ & $4(10)$ & $2(13)$ & $O(0)$ & $2(13)$ \\
\hline Medical school faculty appointment, $n$ (\%) & $20(48)$ & $9(60)$ & $5(42)$ & $6(40)$ \\
\hline
\end{tabular}

*One respondent practiced at both a VA Medical Center and a community-based outpatient clinic

access to one. In fact, only one physician with access to a PDMP indicated that he did not use it (Table 2). One physician from Pennsylvania said, "I would absolutely use it...it would help me cut down on the risk of a patient obtaining opiates from another source, and probably decrease the overall rate of overdose in Pennsylvania."

While physicians endorsed PDMP use in general, they exhibited varying approaches to use. In examining the coded statements from participants, we grouped responses into two categories regarding participants' overarching approach to PDMP use: (1) systematic and (2) subjective. These approaches, which physicians often applied concurrently, were influenced by a combination of state and institutional policies, as well as physicians' concerns for misuse, work flow, and clinic routine. For example, physicians could be classified as applying both approaches if they used the PDMP in accordance with state mandates and when they had clinical suspicion for opioid misuse.

The systematic approach was characterized by algorithmic use of the PDMP, driven largely by state- and facility-level mandates. Physicians who applied this approach routinely checked the PDMP when writing new prescriptions and/or at set intervals thereafter. In Massachusetts, 10/12 (83\%) physicians applied a systematic approach, whereas in Illinois, 11/15 (73\%) physicians applied such an approach. In Pennsylvania, $4 / 15$ (27\%) physicians envisioned applying a systematic approach. One physician from Massachusetts said, "We're required to access the database before we start a patient on chronic opioids, and then periodically we review it for renewals, but that's sort of maybe once or twice a year."

The subjective approach was largely characterized by use of the PDMP when there was clinical suspicion for opioid misuse. Additionally, some respondents indicated that their use was dictated by time and competing demands. Physicians from Illinois $(12 / 15,80 \%)$ were slightly more likely than physicians from Massachusetts $(9 / 12,75 \%)$ to engage in subjective use, while the majority of Pennsylvania physicians $(9 / 15,60 \%)$ envisioned applying a subjective approach. One physician from Illinois said, "So if there are any (red) flags or concerns for me, like if I have a patient who is requesting early refills, or who has lost medications, or I have any other reason to be worried about their risk for... regarding opioids....I use it more for indications rather than just routine review of patients."

Challenge to Underlying Biases. PDMP use also challenged physicians' underlying biases regarding opioid misuse. Overall, five physicians were surprised by certain patients who they discovered were receiving opioids from non-VA prescribers, causing them to re-evaluate their own biases regarding who they suspected could be misusing opioids. One physician said, "I used to kind of make a value judgement of my patients about whether or not I felt they were reliable and make prescribing choices based upon that. And in doing this [using the PDMP] I've realized that all of those things have to go out the window."

\section{Barriers to PDMP Use}

Administrative Burdens. Many physicians felt "nickel-anddimed" regarding the extra time it took to log on to the PDMP, execute a search, and document the findings. One physician commented, "I think it's just the extra step. You have to sign in, you know, and it takes those extra minutes that's hard to find when you're with a busy practice and have only so much time with the patient that you're seeing at that moment."

Physicians also reported an overall increase in workload related to PDMP use, including required documentation and the additive effect of state-based mandates. According to one physician, "One of the things in Massachusetts that I'm a little bit concerned about is (that) I think we're supposed to check the PDMP every time we prescribe a narcotic to one of our patients, even if it's chronic. And that's just adding a little bit more work to everything."

Incomplete or Inaccessible Data. This theme manifested in three key ways. First, physicians were extremely sensitive to the absence of up-to-date VA data within their states' PDMPs. While VA had started to broadly provide pharmacy data with state PDMPs by 2016, many physicians reported that VA data was still unavailable at the time of these interviews. "There has 
Table 2Supplemental Quotes Characterizing VA Physicians' General Experiences, Barriers to Use, and Facilitators in Using Prescription Drug Monitoring Programs

Physician's general experiences

Broad acceptance

Variable approach to use

- Systematic

- Subjective

Challenge to underlying biases

Administrative burdens

- Time requirement

- Increased work

Incomplete or unavailable data - Incomplete VA data

- Unavailable state-level data

- Access for homeless Veterans

Suggestions for future improvement - Integration with the electronic medical record

- Use of templated note within CPRS

Current features that enhance use

- User-friendly website design

- Ancillary staff access
"It gives me the pertinent information-just the stuff I'm looking for. I am even able to check multiple patient identifiers-because you don't want to call a patient and accuse them of something without making sure it's really them."

"We're obligated to do it-to check it every twenty-eight days for people who are on chronic opioids or check it every time before initiation is our rule here."

"Sometimes we do a run down at the end of the month on all the patients. Sometimes every two months or randomly when patients are getting refills, we just randomly pick one and we look it up."

"It's a very helpful tool and I would say it's surprising sometimes who turns out to be doctor shopping and getting narcotics all over the place."

"I think that the more a clinician practices, the more they understand that... you cannot profile someone who is addicted to opiates... It spans both genders, all age groups, all races, and the more experienced clinicians (are)... not hesitant to question if something doesn't add up and say, 'Well, let's just do a PDMP and check that."

"Yeah, it's just the time. Each query probably takes about three to five minutes. Especially if you turn up something and you have to review."

"Our local facility wants us to document at certain intervals using a specific note title in CPRS that we checked, and what we checked, how often we checked, and what we found. So, separate from any other documentation, we have to create a new CPRS documentation every time we check or for patients receiving these medications."

"I think ultimately what we need is they, the non-VA providers, need to have access to what VA providers are prescribing. That's also a critical piece of information so that they can see what we're doing and we can see what they're doing."

"Yeah, if you -I don't know, it's an Illinois registry program that we access to and then there's a drop down list bar so we can click on number of states, but I don't think you can click on all. So the only thing I am able to click on is either Illinois or Wisconsin. So that will give me-if I click more than three or four then it shows me an error."

"I think that we definitely need to have right patient address, which is sometimes complicated because in VA we have homeless people."

"Create an interface between CPRS and state drug monitoring programs where the state drug monitoring program data would automatically show up in CPRS. That would be the best thing that they could do...but it needs to be done in a way that fits in with provider workflow."

"Create a note like the one my medical director was describing that I have not yet seen or used but sounds very good at pulling information from CPRS, and making it clear when things were done and completed. And I think a reminder for those chronic pain patients who meet certain criteria."

"The state has worked really-Massachusetts has worked really hard to make it a more useful tool. So, I think they've done a couple of things just to make it more user-friendly."

"I've now designated one of my nurses to be a surrogate... so that sort of takes that administrative step away from me and will actually allow more of my patients to be checked regularly."

VA Department of Veterans Affairs, PDMP prescription drug monitoring program, CPRS computerized patient record system

been an embarrassing, egregious delay in VA getting access of their data - pharmacy data - to the state's PDMP," said one physician. Physicians felt that this placed extra burden on them to monitor their patients' comprehensive opioid use, as only they had access to their patients' complete record of VA and non-VA prescriptions.

Second, VA physicians consistently commented on differences in the accessibility of neighboring state data within their PDMP. VA physicians in Illinois had access to data from several neighboring states, which they felt enhanced the reach and effectiveness of their PDMP. Despite their close geographic proximity to neighboring states, physicians in Massachusetts did not have access to other states' PDMP data. "It (the PDMP) only gives me information about Massachusetts. So, for our patients who might be accessing medications in bordering states - I have no idea if that's happening or not, and I wish I did."
Third, physicians commented on the difficulty of using the PDMP for homeless Veterans, as one state's PDMP required residential information that was unavailable for homeless Veterans. "I just had one new... patient who ... came to us as homeless. It was difficult for me to check on the prescription monitoring program because they're homeless and we need to use zip code for this (accessing the PDMP)."

\section{Facilitators of PDMP Use}

Suggestions for Future Improvement. In addition to barriers, physicians cited multiple facilitators of PDMP use, including several practices that they recommended states or VA consider to improve use, and those that are currently in place at their VA facilities.

Physicians indicated two key ways that VA could further improve PDMP use. First, physicians frequently commented 
on the importance of integrating data contained within the PDMP with the data and medical records already present within CPRS. One physician commented, "I just think it would be so wonderful if it (PDMP data) could be integrated into our data- our normal accessing of the chart in a way that-If I could just somehow click in the chart and it would fill out a bunch of fields for me - that would be amazing."

Second, physicians indicated that a pre-designed note template within VA's Computerized Patient Record System (CPRS) would expedite documentation and serve as a valuable reference for future clinical encounters. One physician indicated that his facility is currently implementing such a note, while others desired the use of such a note. "When you check the state PDMP, you put the information into (a) templated note that's identifiable in CPRS...The note will automatically populate with the data that the state PDMP was last checked... I wouldn't have to search to find out whether the database has been checked recently or not because it tells me that."

Current Features or Practices that Enhance Use. Physicians felt that the quality of the PDMP website, including the registration process, log on time, and website design, were major factors that enhanced use. One physician said, "The website itself is great. It gives me the pertinent information - just the stuff I'm looking for."

Physicians also valued the ability to delegate responsibility to check the PDMP to nurses and clinical pharmacists in their patient-aligned care teams to decrease their overall work load and facilitate patient care. "The big innovation is that I can delegate authority to query the database to my nurse... I-it's not like it requires all that many keystrokes to get in, but I just wouldn't do it with any regularity if she didn't help."

\section{DISCUSSION}

VA primary care physicians in our study broadly embraced PDMPs as a tool to monitor Veterans' receipt of opioids from non-VA sources despite identifying multiple barriers to optimal use. While the VA physicians in our sample exhibited heterogeneity in their use of PDMPs, they identified several key best practices currently implemented within VA and made suggestions for future improvements that have the potential to enhance efforts to ensure safe opioid prescribing both in VA and non-VA healthcare systems.

While PDMPs represent a potentially powerful tool to monitor Veterans' use of opioids from non-VA sources, our findings suggest that more work needs to be done by VA and at the state level to enhance their use. Physicians in our study consistently indicated that real-time access to prescribing data from both VA and neighboring states would greatly enhance the utility of PDMPs to manage opioid use. While VA was sharing data from most facilities by 2016, respondents in our study consistently noted the lack of up-to-date VA data contained within their state's PDMP. This may reflect their experiences using the PDMP prior to 2016, when less than $50 \%$ of VA facilities were sharing data, ongoing deficiencies in the transmission of pharmacy records, or delays at the state level in incorporating VA data into their respective PDMPs. Such incomplete data places a unique burden on VA providers to monitor the dual receipt of opioids, as non-VA providers cannot account for VA medications when prescribing an opioid. As VA expands and refines its data sharing of pharmacy records, this may enable non-VA providers - if they check the PDMP - to fully account for Veterans' use of opioids and decrease the risk that a Veteran will receive a potentially unsafe opioid prescription both from within or outside VA.

As the largest integrated healthcare system in the USA, ${ }^{29}$ VA may serve as a national model for other healthcare systems and states tasked with optimizing and better integrating PDMP use into everyday practice. Prior studies demonstrate that states that mandate PDMP use and ensure that their PDMP contains up-to-date prescribing data have exhibited greater declines in opioid-related overdose and death as compared to states with less stringent PDMP use requirements..$^{16,30,31}$ This knowledge, along with the fact that physicians in our study reported having biases regarding which patients may be misusing opioids and having those biases challenged after viewing PDMP search results, suggests that mandated systematic use of the PDMP is likely necessary to improve outcomes. While mandated use of the PDMP may be onerous, interviewed physicians identified practices with the potential to overcome these administrative burdens, such as enhanced integration of PDMP data into the electronic medical record (EMR) and enabling ancillary staff to conduct routine PDMP checks. These suggestions are consistent with efforts already being explored by some states. ${ }^{32}$ As both VA and non-VA physicians experience similar barriers to optimal PDMP use, ${ }^{17-22}$ the best practices and suggestions for PDMP improvement we have identified may be broadly applicable in both VA and non-VA healthcare systems.

Our study has important limitations. First, we relied on qualitative methods to establish VA physicians' perspectives and experiences regarding PDMP use. While this approach enabled us to probe deeper into physicians' perceptions regarding PDMPs, this limits generalizability. Additionally, we do not have demographic data on both participants and nonparticipants; therefore, it is possible that those VA physicians who participated in our study are different from those who declined to participate. Nevertheless, our study participants represented a diverse array of sociodemographic and practice characteristics. Second, this study focused largely on structural barriers and facilitators regarding PDMP use, rather than whether PDMP use directly influenced physicians' clinical decision-making. Nevertheless, our findings are timely and may provide VA leadership with valuable data to tailor and maximize the impact of policies and may inform additional quantitative evaluations to enhance PDMP use both within and outside VA. Third, our findings represent the perspectives 
of primary care physicians. Other types of prescribers, such as specialty physicians, dentists, and advanced practice providers, may possess different insights that we did not capture in our interviews. Fourth, we were only able to recruit 12 physicians in Massachusetts rather than the 15 planned, despite reaching out to all eligible VA primary care physicians. Despite the smaller number from this state, our overall sample size was sufficient to achieve thematic saturation.

\section{CONCLUSION}

VA has improved the safe prescribing of opioids among Veterans, ${ }^{4}$ yet these efforts have not directly addressed the significant number of opioid prescriptions that Veterans continue to receive outside VA. Our findings suggest that VA physicians broadly support the use of PMDPs and that with improvements PDMPs have the potential to effectively mitigate Veterans' use of opioids from VA and non-VA prescribers. Applying the best practices and suggestions for improvements we have identified to ongoing efforts to implement PDMP use within VA may enable VA to serve as a national model for states and other institutions who seek to enhance PDMP use and improve the safe prescribing of opioids.

\section{Acknowledgements:}

We would like to acknowledge Peter Glassman, M.B.B.S. and Scott Herrle, M.D., M.S. for their assistance in pilot testing the interview script.

Prior Presentations: This paper has been presented at the Society of General Internal Medicine National Meeting in Washington, D.C. in April, 2017, and at the Academy Health National Meeting in New Orleans, LA in June 2017.

Corresponding Author: Thomas R. Radomski, MD, MS; Center for Research on Healthcare, Pittsburgh, PA, USA (e-mail: radomskitr@upmc.edu).

Funding This project was funded by the VA Health Services Research and Development Merit Review Award (IIR 14-297). Dr. Radomski received support from the National Center for Advancing Translational Sciences of the National Institutes of Health under Award Number KL2TR001856. The content is solely the responsibility of the authors and does not necessarily represent the official views of the National Institutes of Health, the Department of Veterans Affairs, or the US Government.

\section{Compliance with Ethical Standards:}

The final interview script and study protocol were approved by the Institutional Review Board of the VA Pittsburgh Healthcare System. Participants were consented to be audio-recorded prior to the interviews.

Conflict of Interest: The authors declare that they do not have a conflict of interest.

\section{REFERENCES}

1. Opioid Overdose. Available at https://www.cdc.gov/drugoverdose/epidemic/index.html. Accessed on May 3, 2017.
2. Overdose Death Rates. Available at https://www.drugabuse.gov/relatedtopics/trends-statistics/overdose-death-rates. Accessed on May 3, 2017.

3. 10 Leading Causes of Injury Deaths by Age Group Highlighting Unintentional Injury Deaths, United States-2010. Available at https:// www.cdc.gov/injury/wisqars/pdf/10LCID_Unintentional_Deaths_2010a.pdf. Accessed on May 3, 2017.

4. Gellad WF, Good CB, Shulkin DJ. Addressing the Opioid Epidemic in the United States: Lessons from the Department of Veterans Affairs. JAMA Intern Med. 2017; 177(5):611-612.

5. Gellad WF, Zhao X, Thorpe CT, Thorpe JM, Sileanu FE, Cashy JP, Mor M, Hale J, Radomski TR, Hausmann LRM, Fine MJ, Good CB. Overlapping Buprenorphine, Opioid, and Benzodiazepine Prescriptions among Veterans Dually Enrolled in VA and Medicare Part D. Substance Abuse. 2017; 38(1):22-25.

6. Gellad WF, Thorpe JM, Zhao X, Thorpe CT, Sileanu FE, Cashy JP, Hale J, Mor M, Radomski TR, Hausmann LRM, Donohue JM, Gordon AJ, Suda KJ, Stroupe KT, Hanlon JT, Cunningham FE, Good CB, Fine MJ. Impact of dual use of va and medicare part d drug benefits on potentially unsafe opioid use. Am J Public Health. 2018; 108(2):248-255.

7. Suda KJ, Smith BM, Bailey L, Gellad WF, Huo Z, Burk M, Cunningham FE, Stroupe KT. Opioid dispensing and overlap in veterans with non-cancer pain eligible for medicare part D. J Am Pharm Assoc.2017;57:333-340.

8. Gellad WF, Cunningham FE, Good CB, Thorpe JM, Thorpe CT, Bair B, Roman K, Zickmund SL. Pharmacy use in the first year of the veterans choice program: a mixed-methods evaluation. Med Care.. 2017; 55 Suppl 7 Suppl1:S26-S32.

9. Shulkin DJ. Beyond the VA crisis-becoming a high-performance network. N Engl J Med.2016;374:1003-1005.

10. U.S. Department of Veterans Affairs. 2011 Survey of Veteran Enrollees' Health and Reliance Upon VA. Available at: http://www.va.gov/ healthpolicyplanning/soe2011/soe2011_report.pdf. Accessed on 24 May 2015.

11. Gasper J, Liu H, Kim S, May L. 2015 Survey of veteran enrollee's health and use of health care. http://www.va.gov/HEALTHPOLICYPLANNING/ SoE2015/2015_VHA_SoE_Full_Findings_Report.pdf. Accessed 31 March 2017.

12. Kizer KW. Veterans and the affordable care act. JAMA. 2012;307(8):78990.

13. Radomski TR, Zhao X, Thorpe CT, Thorpe JM, Good CB, Mor MK, Fine MJ, Gellad WF. VA and medicare utilization among dually enrolled veterans with type 2 diabetes: a latent class analysis. $J$ Gen Intern Med.2016;31(5):524-31.

14. Reisman RM, Shenoy PJ, Atherly AJ, Flowers CR. Prescription opioid usage and abuse relationships: an evaluation of state prescritpion drug monitoring program efficacy. Subst Abuse.2009;3:41-51.

15. Status of Prescription Drug Monitoring Programs (PDMPs). Available at http://www.pdmpassist.org/pdf/PDMPProgramStatus.pdf. Accessed on May 3, 2017.

16. Weiner J, Bao $\mathbf{Y}$, Meisel Z. Prescription drug monitoring programs: evolution and evidence. Available at https://ldi.upenn.edu/brief/prescription-drug-monitoring-programs-evolution-and-evidence. Accessed on June 29, 2017.

17. Blum CJ, Nelson LS, Hoffman RS. A survey of Physicians' Perspectives on the New York State Mandatory Prescription Monitoring Program (ISTOP). J Subst Abuse Treat. 2016;70:35-43.

18. Rutkow L, Turner L, Lucas E, Hwang C, Alexander GC. Most primary care physicians are aware of prescription drug monitoring programs, but many find the data difficult to access. Health Aff (Millwood). 2015;34(3):484-92.

19. Lin DH, Lucas E, Murimi IB, Jackson K, Baier M, Frattaroli S, et al. Physician attitudes and experiences with Maryland's Prescription Drug Monitoring Program (PDMP). Addiction. 2016.

20. Carnes NA, Wright ER, Norwood CW. A qualitative analysis of prescribers' and dispensers' views on improving prescription drug monitoring programs. Res Social Adm Pharm. 2016.

21. Hildebran C, Cohen DJ, Irvine JM, Foley C, O'Kane N, Beran T, et al. How clinicians use prescription drug monitoring programs: a qualitative inquiry. Pain Med. 2014;15(7):1179-86.

22. Irvine JM, Hallvik SE, Hildebran C, Marino M, Beran T, Deyo RA. Who uses a prescription drug monitoring program and how? insights from a statewide survey of Oregon clinicians. J Pain. 2014;15(7):747-55.

23. Comprehensive Addiction and Recovery Act of 2016, 42 U.S.C. $\S \S 114-$ 198. 2016.

24. Consolidated Appropriations Act of 2016 
25. Sandelowski M. Focus on qualitative methods: sample size in qualitative research. Res Nurs Health. 1995; 18:179-183.

26. Miller W, Crabtree BF. Primary care research: a multi typology and qualitative road map. In: Crabtree BF, Miller WL, eds. Doing qualitative research. London: Sage Press, 1992. pp 3-30.

27. Barnett JM. Review Note: Benjamin Crabtree \& William Miller (Eds.) 2002 .

28. Doing Qualitative Research (2nd ed.) [20 paragraphs]. Forum Qualitative Sozialforschung / Forum: Qualitative Social Research [On-line Journal], 3(4). Available at: http://www.qualitativeresearch.net/fqs/fqs-eng.htm.

29. Providing Health Care for Veterans. Available at https://www.va.gov/ health/. Last accessed on June 6, 2017.
30. Briefing on PDMP Effectiveness. PDMP Center of Excellence at Brandeis. Available at http://www.pdmpassist.org/pdf/COE_documents/Add_to_ TTAC/Briefing\%20on\%20PDMP\%20Effectiveness\%203rd\%20revision. pdf. Accessed on May 9, 2017.

31. Pardo B. Do more robust prescription drug monitoring programs reduce prescription opioid overdose? Addiction. 2016. https://doi.org/10.1111/ add.13741.

32. Reilly C. Improvements to prescription drug monitoring programs can help stop overdose deaths. Available at http://www.pewtrusts.org/en/ research-and-analysis/analysis/2016/02/11/improvements-to-prescription-drug-monitoring-programs-can-help-stop-overdose-deaths. Last Accessed on July 3, 2017. 